\title{
From non-human to human: Adult's and children's perceptions of agents varying in humanness
}

\author{
Eva Krumhuber ${ }^{1}$, Arvid Kappas ${ }^{2}$, Colette Hume ${ }^{3}$, Lynne Hall ${ }^{3}$, Ruth Aylett ${ }^{4}$ \\ ${ }^{1}$ University College London, London, WC1H 0AP, UK \\ ${ }^{2}$ Jacobs University Bremen, 28759 Bremen, Germany \\ ${ }^{3}$ University of Sunderland, Sunderland, SR6 0DD, UK \\ ${ }^{4}$ Heriot-Watt University, Edinburgh, EH14 4AS, UK \\ e.krumhuber@ucl.ac.uk \\ a.kappas@jacobs-university.de \\ \{colette.hume, lynne.hall\}@ sunderland.ac.uk \\ ruth@macs.hw.ac.uk
}

\begin{abstract}
While most interface agents have been designed from an adult perspective, the present paper compares adults' and children's views of agents that vary in their degree of humanness. Four synthetic characters ranging in appearance from non-human to very human (blob, cat, cartoon, human) were presented to adult and children perceivers and were evaluated with respect to their cognitive and emotional abilities. The visual appearance significantly influenced participants' ratings in both age groups. However, the pattern of results was more differentiated for adult perceivers as a function of the humanlikeness of the character. The findings suggest that children may rely less on human-like features in inferring agents' capabilities which are judged along simpler cognitive and social dimensions. Implications for the design of artificial agents are discussed.
\end{abstract}

Keywords: agent, appearance, human-like, theory of mind, children

\section{Introduction}

Current evidence suggests that users prefer and rely more on human-like agents/robots than mechanical-looking or abstract visual representations [1, 2]. While human-like characteristics contribute to a more human perception, there is one dimension of human mind that is seen as unique to humans: self-conscious mental experience [3]. The experience of mind with complex emotions and abstract thought appears to be reserved only for humans. This is supported by evidence showing that people rarely give moral rights and privileges to machines such as robots [4]. But does this apply to everyone and of every age? The goal of the present research was to examine how adults and children infer human qualities of virtual characters that vary in appearance from nonhuman to very human. To elucidate this question, we selected attributes that target dispositional traits, mental states, as well as basic and complex emotions. Whereas simple traits and emotions (e.g., likeable, trustworthy, angry) may be easily attributed to mechanical and animal looking characters, abstract concepts such as mind and shame 
require more cognitive complexity than what might be apparent on first sight $[5,6,7]$. Based on previous research $[8,9]$ we predicted that the degree of human-likeness would significantly affect adults' attributions as to the agent's mental and emotional capabilities. These effects were expected to be less pronounced for children who might judge the characters along simpler cognitive and social dimensions.

\section{Experiment}

40 adults $\left(M_{\text {Age }}=20.33\right)$ and 35 children $\left(M_{A g e}=10.06\right)$ were presented with either static or dynamic displays of four embodied characters that differed in their degree of human-likeness: blob, cat, cartoon, and human (see Figure 1). All characters and animations were created in 3ds Max using a default biped and were displayed on blue background (490 × 270 pixels). After viewing each stimulus participants answered the following questions on 7-point Likert-scales ranging from (1) not at all to (7) very much: a) How likeable is the character?, b) How trustworthy is the character?, c) How intelligent is the character?, d) How engaging is the character?, e) To what degree does the character have a mind on its own?, f) To what degree can the character experience anger?,g) To what degree can the character experience shame? These questions were posed in random order, with one question per stimulus presentation.

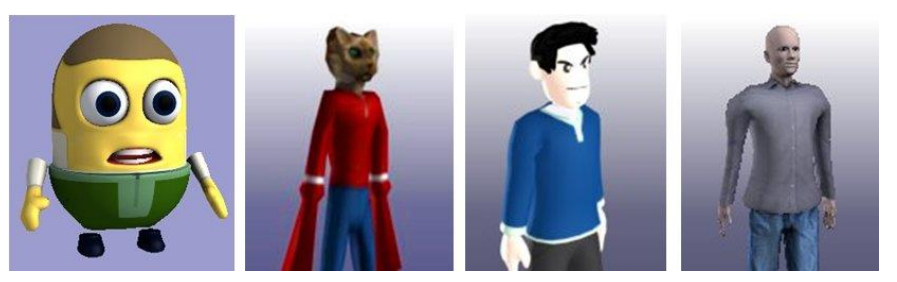

Fig. 1. Four embodied characters - blob, cat, cartoon, human - from non-human to very human in a neutral position.

\section{Results}

A multivariate analysis of variance (MANOVA) revealed a significant interaction between Age Group and Stimulus Character, $F(21,47)=4.50, p<.001, \eta_{p}{ }^{2}=.67$. In univariate terms, this interaction was significant for almost all variables: likeable, $p=$ .009 , trustworthy, $p=.006$, intelligent, $p=.050$, engaging, $p=.214$, mind, $p<.001$, anger, $p=.014$, and shame, $p=.091$.

As can be seen in Figure 2, children's ratings were generally higher than those of adults. This was particularly the case for the blob which was judged by children as more trustworthy, intelligent, likely to have a mind, and capable of experiencing anger and shame $(p s<.05)$. Similarly, children made higher attributions of mind, anger and shame when evaluating the cat $(p s \leq .05)$. Differences between adults and children also occurred for the cartoon character which was seen by children as more likeable, trustworthy, and capable of experiencing shame $(p s<.01)$. For the human character, 
children' ratings differed from those of adults only for anger $(p<.01)$, with higher scores given by children.

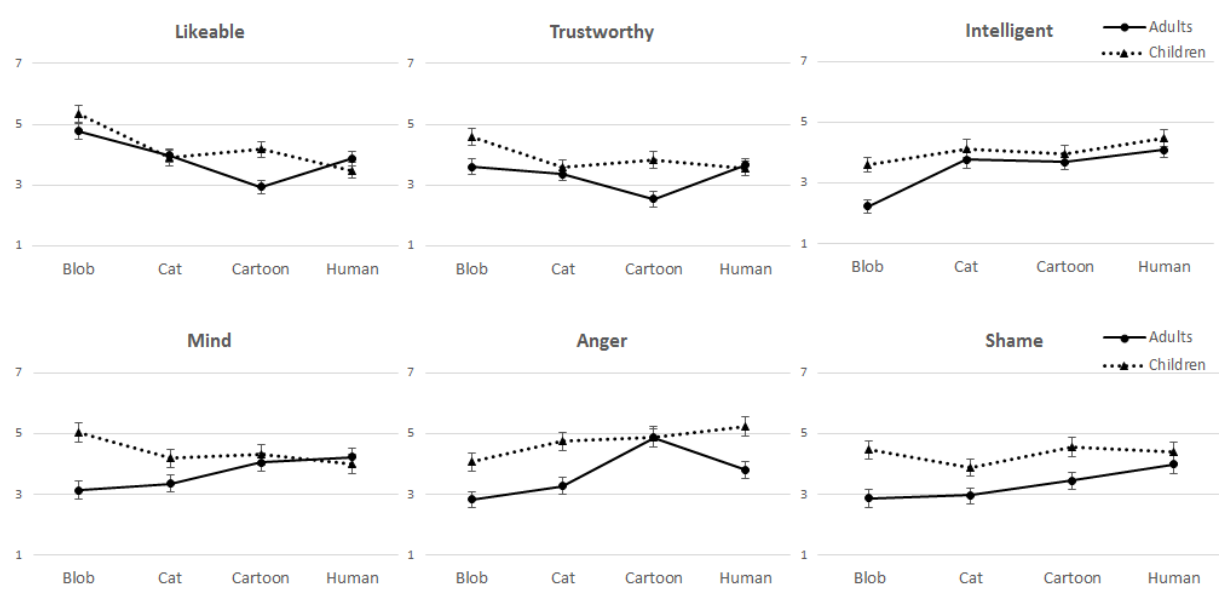

Fig. 2. Adults' and children's mean ratings of the four characters. Error bars represent standard errors.

\section{Discussion}

As shown in the present study, adults attributed complex states, such as mind and shame to a greater extent to characters that were at the endpoint of the human scale (i.e., cartoon, human). These findings are in line with previous research [1], [10, 11, 12, 13] and suggest that the choice of visual representation drives attributions regarding the cognitive and emotional intelligence the system invites. Given that such effects are fast, in part automatic, and might not habituate over short exposures, the design of applications should therefore take a match between the perceived profile of the agents and the intended function into account. Although children made consistent and generally higher attributions regarding intellectual and emotional capabilities, a distinction was largely apparent only between the characters at the endpoints of the artificial/human scale (i.e., blob, human). Among all visual forms, the blob was most preferred and this favourable impression generalized across other characteristics (i.e., mind, trustworthiness). In accordance with previous research, for children a human-like appearance may therefore not be a direct criterion for inferring the agents' abilities [e.g., $5,6]$.

In this research we have shown that assessing adults' and children's views can be essential for the design of embodied visual forms in human-computer interaction. Up to now, most guidelines for building effective interface agents are based solely on adult perspectives, thereby overlooking children's social and cognitive requirements. Here a closer collaboration of psychologists and computer scientists and engineers can be 
particularly promising. We do not believe that agent design can be a "one-size fits all" affair. Instead, agents, tasks and users must be carefully matched to achieve an optimal interaction between humans and artificial systems.

Acknowledgments. This work has been conducted within the European Commission project eCUTE - Education in Cultural Understanding, Technologically-Enhanced (FP7-ICT-2009.4.2). We thank Tony Manstead for his help with data collection and Nadia Malas for editorial work.

\section{References}

1. Goetz, J., Kiesler, S., Powers, A.: Matching Robot Appearance and Behavior to Tasks to Improve Human-Robot Cooperation. In: Proceedings of The IEEE International Workshop on Robot and Human Interactive Communication, pp. 55-60. Milbrae, California (2003).

2. Hinds, P. J., Roberts, T. L., Jones, H.: Whose Job is It Anyway? A Study of Human-Robot Interaction in a Collaborative Task. Hum-Comput. Interact, 19, 151-181 (2004).

3. Gray, H. M., Gray, K., Wegner, D. M.: Dimensions of Mind Perception. Science. 315, 619 (2007).

4. Friedman, B., Kahn, P. H. Jr., Hagman, J.: What Online AIBO Discussion Forums Reveal About the Human-Robotic Relationship. In: Proceedings of the Computer-Human Interaction Conference CHI, pp. 273-280, New York (2003).

5. Hall, L., Woods, S., Dautenhahn, K., Sobral, D., Paiva, A., Wolke, D., Newall, L.: Designing Empathic Agents: Adults vs. kids. Lect. Notes Comput. Sc. 3220, 604-613 (2004b).

6. Paiva, A., Dias, J., Sobral, D., Aylett, R., Woods, S., Hall, L., Zoll, C.: Learning By Feeling: Evoking Empathy With Synthetic Characters. Appl. Artif. Intell. 19, 235-266 (2005).

7. Bumby, K. E., Dautenhahn, K.: Investigating Children's Attitudes Towards Robots: A Case Study. In: Proceedings of the CT99: The Third Cognitive Technology Conference. San Francisco (1999).

8. Woods, S., Dautenhahn, K., Schulz, J.: The Design Space of Robots: Investigating Children's Views. In: Proceedings of the IEEE International Workshop on Robot and Human Interactive Communication, pp. 47-52, Kurashiki (2004).

9. De Melo, C., Carnevale, P., Gratch, J.: Humans vs. Computers: Impact of Emotion Expressions on People's Decision Making. In: IEEE Transactions on Affective Computing (in press).

10. Parise, S., Kiesler, S., Sproull, L., Waters, K.: My Partner is a Real Dog: Cooperation With Social Agents. In: Proceedings of the ACM Conference on Computer Supported Cooperative Work (CSCW), pp. 399-408, New York (1996).

11. Koda, T., Maes, P.: Agents With Faces: The Effects of Personification of Agents. In: Proceedings of the $5^{\text {th }}$ IEEE International Workshop on Robot and Human Communication RO-MAN'96, pp. 189-194, Tsukuba (1996).

12. Hegel, F., Krach, S., Kircher, T., Wrede, B., Sagerer, G.: Theory of Mind (ToM) on Robots: A Functional Neuroimaging Study. In: Proceedings of the Human-Computer Interaction Conference HRI, pp. 335-342, Amsterdam (2008).

13. Harris, P. L, Donnelly, K., Guz, G. R., Pitt-Watson, R.: Children's Understanding of the Distinction Between Real and Apparent Emotion. Child Dev. 57, 895-909 (1986). 